\title{
Calibration, Performance, and Cosmic Ray Detection of ARIANNA-HCR Prototype Station
}

\section{Shih-Hao Wang* for the TAROGE collaboration and the ARIANNA collaboration}

National Taiwan University, No. 1 Sec. 4, Roosevelt Rd., Taipei 10617, Taiwan

${ }^{*}$ E-mail: wsh4180@gmail.com

\begin{abstract}
ARIANNA Horizontal Cosmic Ray (HCR) station is an antenna array aiming at detecting RF impulses emitted from nearly horizontal extensive air showers (EAS) generated by cosmic rays above about $0.1 \mathrm{EeV}$, and ultra-high energy Earth-skimming $v_{\tau}$ coming out from nearby mountains. A prototype station consisting of four log-periodic dipole antennas above the ice, in frequency range of $140-500 \mathrm{MHz}$, is deployed at Ross Ice Shelf in Antarctica during December, 2016. In this paper, the instrumentation of ARIANNA-HCR and the data set collected are presented. Next, the calibration using both ground-based and balloon-borne transmitters and the resulting timing and angular resolution are presented. Then the first result on the search of cosmicray EAS candidates and their angular distribution are reported. Finally, we present the expansion plan of ARIANNA-HCR station.
\end{abstract}

35th International Cosmic Ray Conference

10-20 July, 2017

Bexco, Busan, Korea

${ }^{*}$ Speaker. 


\section{Introduction}

Radio detection of extensive air showers (EAS) induced by high energy cosmic rays above $10^{16} \mathrm{eV}$ has been established in the past decade and shown to be competitive with and complementary to conventional particle, air-fluorescence, and air-Cherenkov approaches (see [1, 2, 3] for reviews). The radio emission of EAS mainly comes from electrons and positrons in the shower being deflected oppositely by geomagnetic field, producing transverse current which radiation is linear polarized along the direction of Lorentz force. The secondary contribution, which accounts for about $10 \%$ of total signal strength, is from Askaryan effect that the shower develops into 10$20 \%$ negative charge excess when interacting with air, leading to radiation radially polarized from the shower axis. The two effects combine and form coherent emission at wavelength longer than longitudinal size of shower front, which can reach about $1 \mathrm{GHz}$, and is forward-beamed with opening angle about $1^{\circ}$ due to relativistic and Cherenkov effect. Recently, observatories such as AERA [4] and LOFAR [5], operating at frequency of 30-80 MHz and using either particle or optical detectors as trigger and for cross calibration, demonstrated that the radiation strength and the depth of shower maximum, which is related to the energy and the composition of primary cosmic ray, respectively, can be precisely measured and confirmed the consistency with simulation. In addition, standalone radio observatories such as ANITA [6] and ARIANNA [7, 8] in Antarctica, which primary goal is detecting UHE neutrinos and at frequency range around $100-1000 \mathrm{MHz}$, have also shown their capability of hunting ultra-high energy (UHE) cosmic rays.

In this paper, we report the first result on the ARIANNA horizontal cosmic ray (HCR) prototype station. The HCR station, collocated with ARIANNA hexagonal radio array (HRA) stations at Ross Ice Shelf in Antarctica, is deployed during December, 2016. It is an on-ice antenna cluster pointing horizontally almost due north toward Mt. Discovery with the goals: 1) monitoring radio-frequency (RF) background above the ice; 2) detecting and characterising inclined cosmicray induced air-showers, complementary to $\mathrm{CR}$ detection at HRA and served as additional veto for UHE neutrino detection; 3) investigating the potential of detecting nearly horizontal air-shower induced by $\tau$-lepton generated by Earth-skimming UHE $v_{\tau}$ interacting with mountains [9].

\section{Instrumentation and Setup}

The overall design of HCR station is the same as HRA stations (see [7, 10] for details) except the antennas as well as their configuration. Four log-periodic dipole antennas (LPDA) sensitive to $140-1000 \mathrm{MHz}$, with gain of 6-8 $\mathrm{dBi}$ and half beamwidth of about $40^{\circ}$, are mounted on a $4.8 \mathrm{~m}$ high tower, with the arrangement and the response as shown in Fig. 1. Three of antennas are horizontally polarized (Hpol) because of nearly vertical local geomagnetic field (inclination $<-80^{\circ}$ ), with one located at the top of the tower about $4.3 \mathrm{~m}$ above the surface. The other two Hpol are located on a horizontal truss that is positioned about $1.5 \mathrm{~m}$ above the surface. They are separated by $1.5 \mathrm{~m}$. A third LPDA on the bottom truss is centered between the two Hpol LPDAs, but vertically polarized (Vpol) for the polarization analysis. The signal is band-pass filtered to 100 $500 \mathrm{MHz}$, and fed into low noise amplifier (LNA) and data-acquisition (DAQ) system which are EMI-shielded in aluminium boxes and below the snow for stable thermal environment. Being in the 

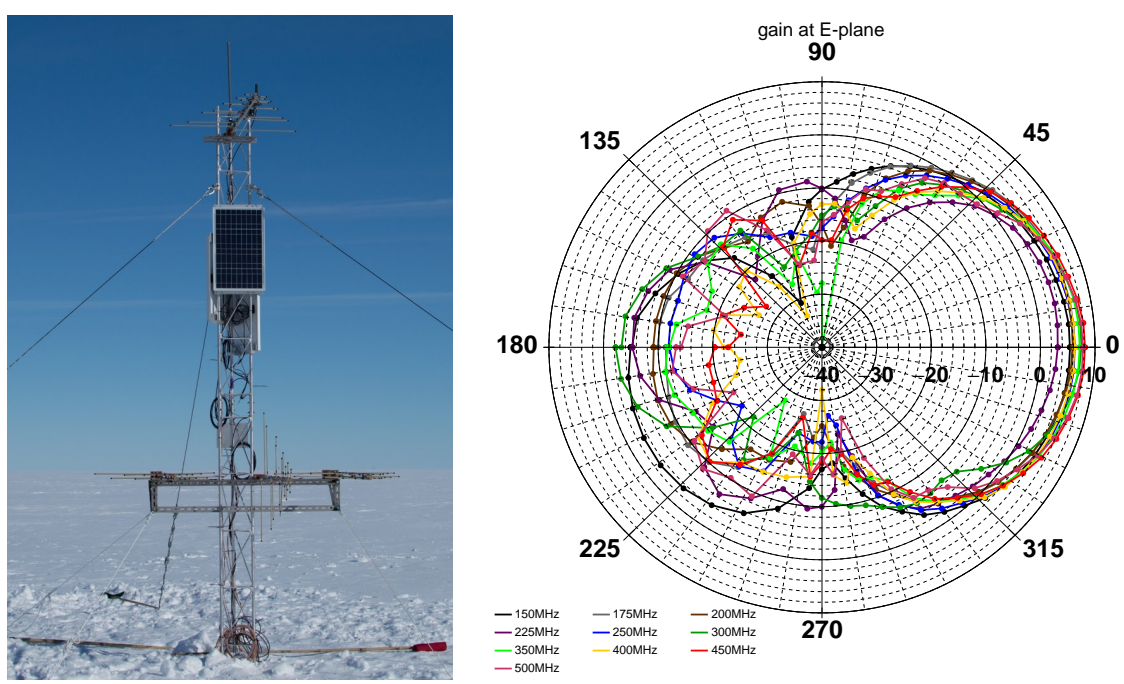

Figure 1: Left: photo of HCR station, where one Hpol LPDA at the top and three at the bottom with (H, V, $\mathrm{H})$ configuration. Right: the gain pattern of LPDA at E-plane (the plane containing antennas elements) in $\mathrm{dBi}$ at several chosen frequencies, measured in anechoic chamber.

isolated area, the entire system is autonomous, and designed to have very low power consumption of $4 \mathrm{~W}$ that is supplied by solar panels and $\mathrm{LiFePO}_{4}$ batteries.

The DAQ is integrated to a single circuit board in which the SST chip [11] takes charge of digitization and trigger. The chip has 4 channels with 256 samples for each, 12-bits dynamic range, and with sampling rate set to $1 \mathrm{GHz}$. After sampling the trigger is processed, where at channel level it requires the signal exceeds dual-sided threshold within a given time window, and two-out-of-four channel coincidence at station level. An additional level 1 (L1) trigger is activated for vetoing narrow band, continuous wave $(\mathrm{CW})$ noises in real time. It computes the ratio of the magnitude at the maximum bin of a spectrum to the sum of the rest, and an event is rejected if the value exceeds a given threshold of 0.3 . To study the RF background, a forced trigger at every 100 seconds is set to obtain unbiased samples. The DAQ system can be remotely configured and monitored via Wi-Fi and Iridium satellite communications. These communication systems transfer data back to a server in the US in nearly real time, except when the sun is no longer capable of supplying reliable power near the end of March.

\section{Station Operation and RF background}

Since its deployment in the late November, 2016, the HCR station acquired data continuously until ran out of power at the end of austral summer around mid April, 2017. Data flow ceased on two occasions: during a 4-day period to transfer data, and another day when the station was put into low power mode that allowed the LNA to cool from an over-temp condition. The available data at the server has estimated live time of 72.5 days, while there are about 14-day data not transferred back yet. The event rate typically ranges from $10^{-3}$ to $1 \mathrm{~Hz}$ in a day, and can be up to order of $10 \mathrm{~Hz}$ during most noisy periods. In general, the on-ice HCR station is noisier than HRAs in ice, because it points to the North where the Galactic center and the Sun can come into field of view 

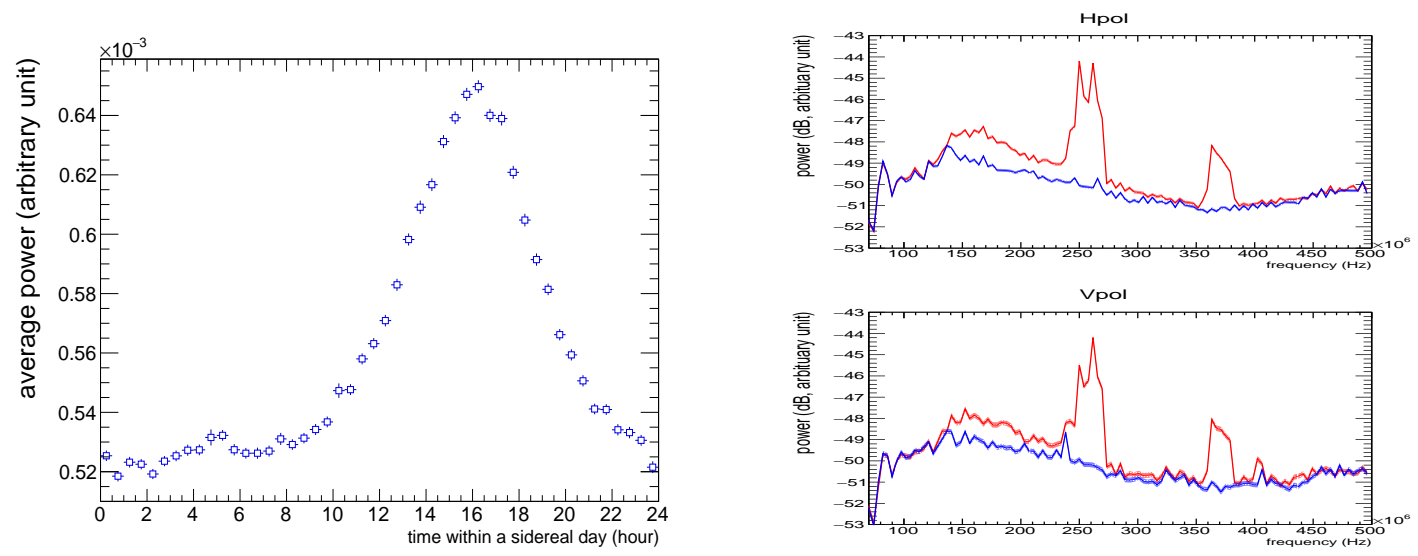

Figure 2: Left: average band power in $140-230 \mathrm{MHz}$ over all channels, versus time expressed in hours in a sidereal day (defining local midnight at 2016-11-15 11:00Z as origin), for all 53658 available forcedtrigger events from 2016-12-13 12:00Z to 2017-04-13 03:00Z. Right: average background spectra at the hour around minimum (blue) and maximum (red) noise level at Hpol (averaged) and Vpol channels.

and McMurdo station just behind the mountains. The variation of noise level with time in lower passband in 140-230 MHz showing a period of one sidereal day (Fig. 2, left), the time reaching the maximum, and the average noise spectra exhibiting power-law like excess during maximum (Fig. 2, right), are all consistent with Galactic origin. As shown in the average spectra, the air-traffic control (ATC) communication around 240-280 MHz and 350-390 MHz is major anthropogenic noise for HCR station, typically having a wave-packet like waveform, and accounting for $>40 \%$ of events, otherwise it shows a clean spectrum with no persistent CW in Hpol channels. Several transient CWs with known sources and frequencies are well suppressed by L1 trigger and can also rejected easily in analysis. The other common type of noise, accounting for $>30 \%$, were correlated with storms (defined as periods of high winds with velocities in excess of $15 \mathrm{~m} / \mathrm{s}$ ), usually appeared in groups within hours or days together with high event rate. These events are characterized by anomalously large signal in only one channel and unusually large spectral power in the stopband. Both features allow high-wind events to be rejected efficiently.

\section{Event Reconstruction and Calibration}

The direction of events is reconstructed with three Hpol antennas (channels) by interferometric method similar to that in [14]. For any pulses transmitted from a distance, these antennas of identical design and at similar observation angle are expected to have similar signal shape. For each antenna pair, two waveforms are cross-correlated with a $140 \mathrm{~ns}$ time window applied around the peak of each to reduce the bias from noise. Given a hypothetical source direction, the time difference of arrival of each pair is calculated according to its baseline, and the correlation coefficient can be looked up given the time delay. The reconstructed direction is chosen at which the sum of coefficients over all pairs reaches maximum. Conversely, if the source direction is known, the relative position of each antenna and internal time delay of each channel can be calibrated.

Two kinds of calibration pulser are used for timing and angular calibration. A ground-based transmitter was set up after the deployment. It was placed about $17 \mathrm{~m}$ away from the station and 
sent pulses from several angles. Since the source is stationary, by analysing the difference of arrival time between two channels, the timing resolution of $50 \mathrm{ps}$ between pairs is derived.

The other source is from HiCal-2 experiment (similar to instrument described in [12]), which consists of two balloon-borne transmitters originally for studying the radio reflectivity and the roughness of Antarctic ice and as in-flight calibration of ANITA-4 [13]. Pulsing at about every 5 $\mathrm{s}$ and having a GPS module recording pulsing time and payload location, both of HiCal-2 flights come into field of view of HCR station not long after their launch, spanning elevation from $2^{\circ}$ to $30^{\circ}$ and azimuth from $-60^{\circ}$ to $35^{\circ}$ (Fig. 3, left), providing valuable calibration data. By comparing the expected angles with the reconstructed ones (described in next paragraph), the absolute orientation of station is corrected, and the angular resolution of $1.4^{\circ}$ in azimuth and $0.6^{\circ}$ in elevation is obtained (Fig. 3, right). There is a systematic offset at reconstructed azimuth when the true angle more negative than $-40^{\circ}$, which is still under investigation but likely caused by the asymmetric antenna response between left and right Hpol LPDA induced by local metal of the tower frame and central Vpol LPDA.
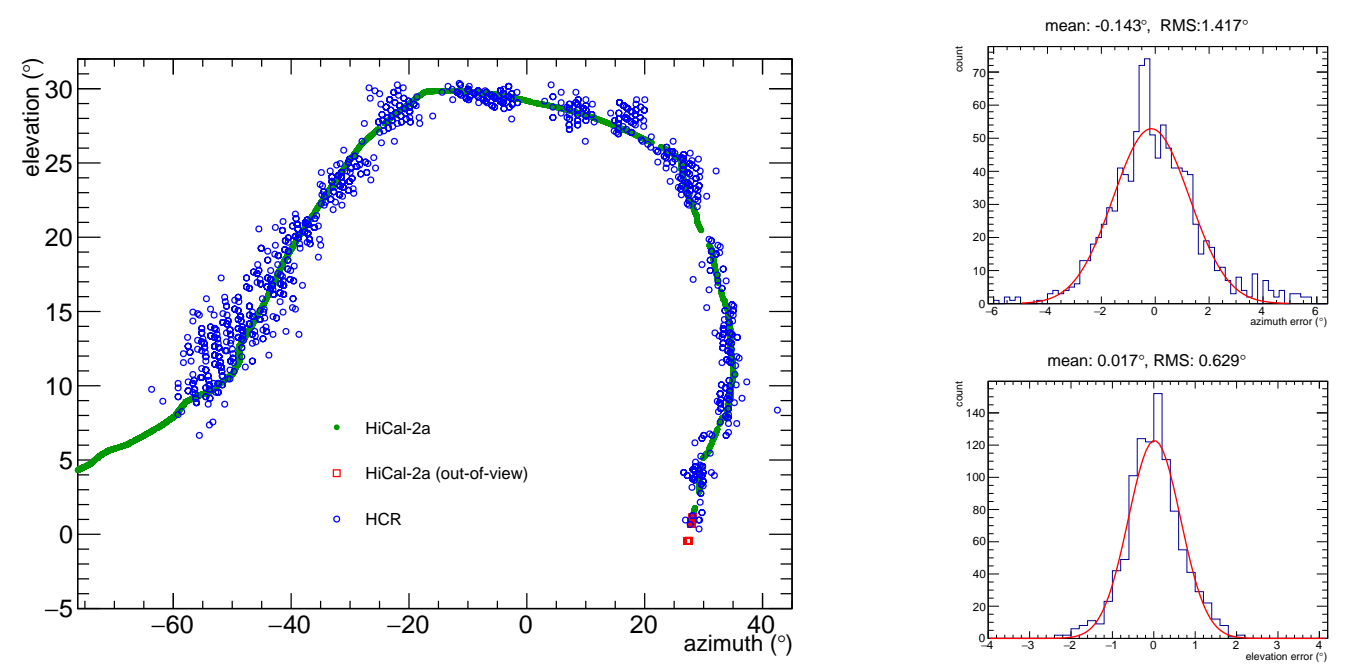

Figure 3: Left: angular map of selected HiCal-2a pulse events: the expected (green tracks), starting from bottom right, and the reconstructed (blue circles). Righ: angular resolution in azimuth (top) and elevation (bottom), selecting events from directions within the main lobe of LPDA and elevation $>10^{\circ}$.

The calibration with $\mathrm{HiCal}$ also reveals that for events below about $10^{\circ}$ elevation, the reconstructed elevation is lower than the true angle, with an error as large as $5^{\circ}$. The unusually large uncertainty is created by interference between the direct signal and a reflected signal from the relatively smooth air-firn surface. The strength of the reflected signal increases rapidly for elevation angles $10^{\circ}$ or less. The relative time delay between the top and bottom LPDAs differs by a few nanoseconds, which affects the waveforms asymmetrically. Consequently, the interference deteriorates the accuracy of the cross-correlation and also affects the measured power. The highly stable environment of the HCR site creates the opportunity to deconvolve the effects of interference to obtain non-interfered waveforms. The procedure is successfully applied to HiCal events to reduce the uncertainty to $1^{\circ}$ for events with elevation angles below $10^{\circ}$. We plan to apply the deconvolution procedure to cosmic-ray events. Correcting reflective interference is important for distinguishing 
very inclined air showers from UHECRs from quasi-horizontal ones originated from $v_{\tau}$.

\section{Cosmic ray search}

A preliminary search for cosmic-ray signals uses data starting from 2016-12-13 12:00Z when both the deployment team has left the field and HiCal-2 is no longer in view. It contains 531627 normal-trigger events during a live time of 62.1 days. Due to large uncertainty in elevation as mentioned in Sec. 4, events with reconstructed elevation $<10^{\circ}$ are removed from this analysis. We describe the additional selection criteria next.

First, the cosmic-ray events should have larger signals in Hpol channels than in Vpol because of dominant geomagnetic effect. Lower passband at $140-230 \mathrm{MHz}$ where the cosmic-ray signal is stronger is used to evaluate the power, and the cut on Hpol to Vpol power ratio larger than $5 \mathrm{~dB}$ is set, which is at $4 \sigma$ level of forced-trigger distribution. This requirement can in principle reject most unpolarized, thermal-like noise and CWs that are not Hpol dominated. Second, in the event reconstruction the plane wave front is assumed for cosmic-ray signal, for the shower maximum is typically at least several kilometers away and the proximity of antennas. Under the approximation, the signal strength at Hpol channels should be similar in contrast to high-wind events, and hence the cut on both peak voltage and power ratios of left versus right and top versus bottom are set. Third, in order to reject ATC and high-wind noise, thresholds on band power ratio between 140$230 \mathrm{MHz}$ to $240-280 \mathrm{MHz}$ and to $60-110 \mathrm{MHz}$ are set, respectively. A modest average correlation coefficient (between $[0,1]$ ) cut on $>0.5$ is set for picking up weaker events. Since cosmic rays above $10^{17} \mathrm{eV}$ have low flux, after applying correlation cut, a time cluster cut is required that a candidate should have no adjacent events within $\pm 100 \mathrm{~s}$.
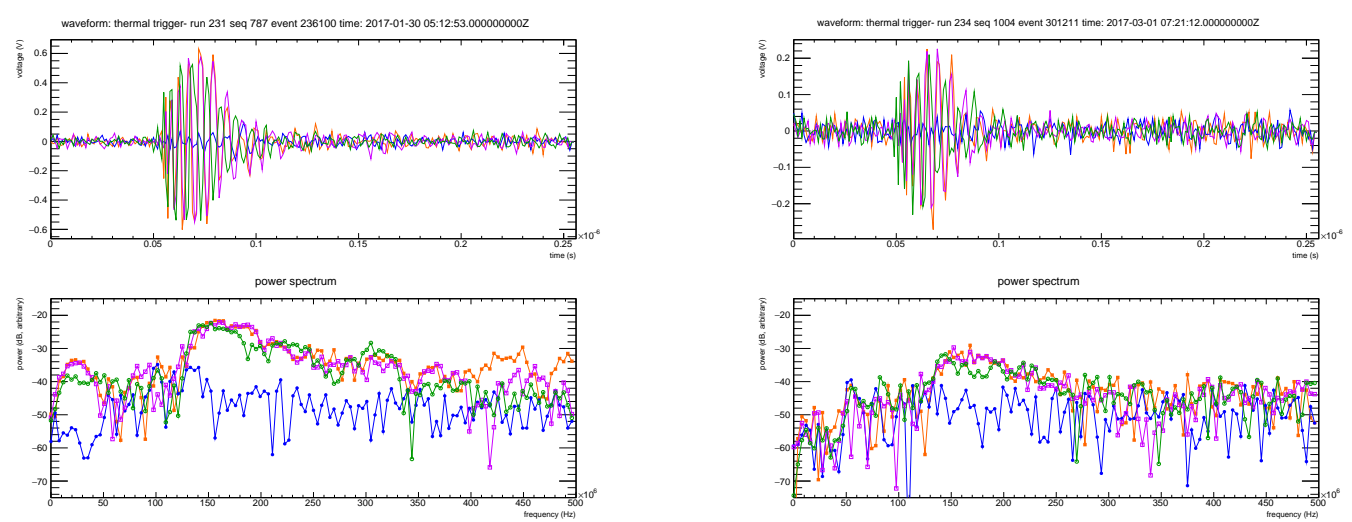

Figure 4: Waveform (top panel) and power spectrum (bottom, power in log-scale and arbitrary unit) of two cosmic-ray candidates with (azimuth, elevation) $=\left(2.5^{\circ}, 23.2^{\circ}\right)$ and $\left(-3.4^{\circ}, 25.6^{\circ}\right)$, respectively, where orange (magenta) for west (east) bottom Hpol channel, green for top Hpol, and blue for Vpol.

After all the cuts are applied, 68 cosmic-ray-like candidates are selected (two examples in Fig. 4), and the resulting distributions of angle, peak voltage, and correlation versus time are shown in Fig. 5 and Fig. 6. Most of candidates fall within the front lobe of LPDAs, and are concentrated in elevation of $15^{\circ}-30^{\circ}$ and in peak voltage of $100-170 \mathrm{mV}$. 

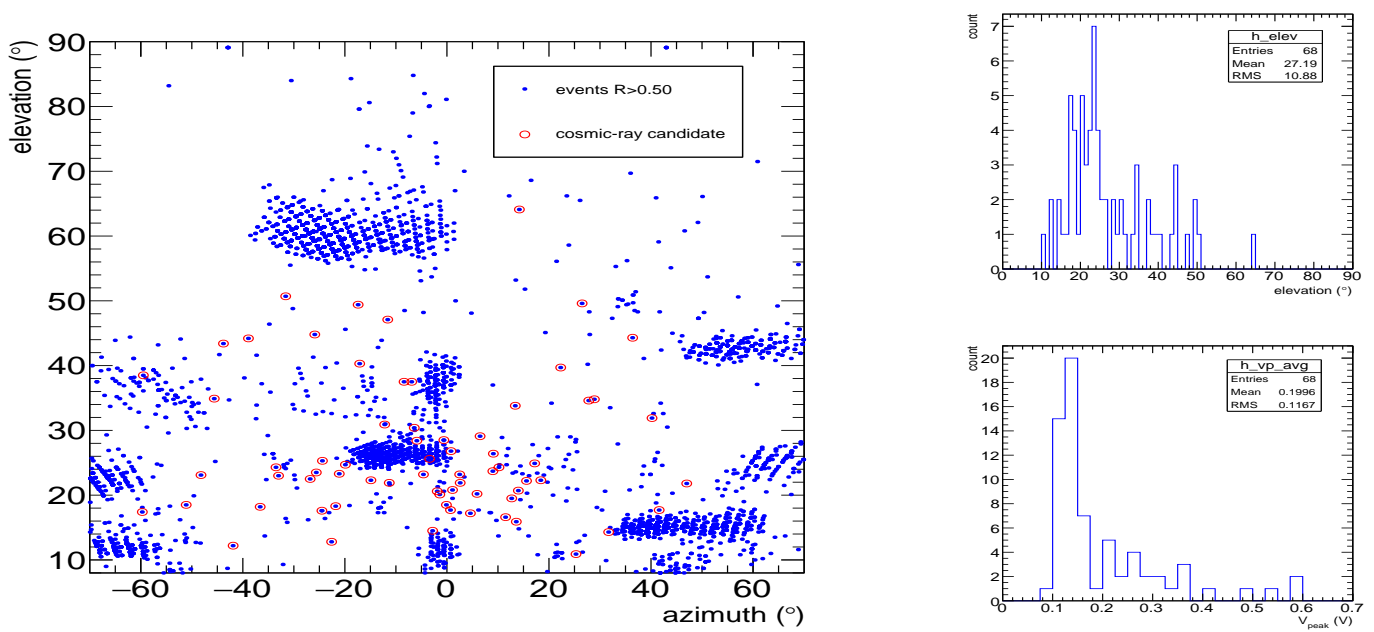

Figure 5: Angular map of 68 cosmic-ray candidates with elevation $>10^{\circ}$ (red circles). Right: distribution of elevation (top) and average Hpol peak voltage (bottom) for cosmic-ray candidates.

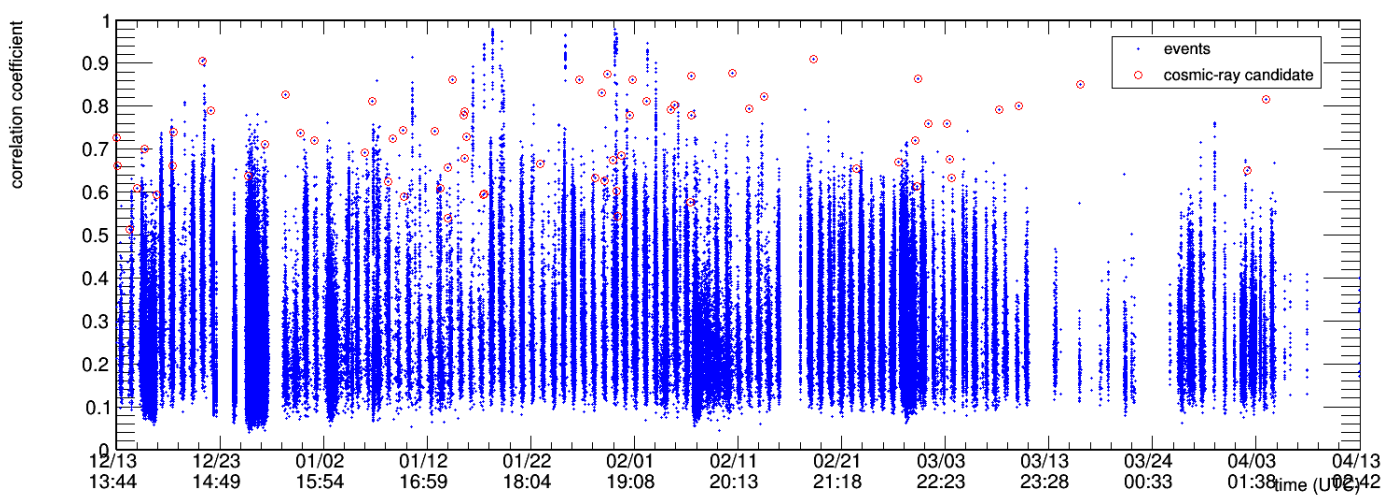

Figure 6: Averaged cross-correlation coefficient of angular reconstruction versus time, where blue dots for all events and red circles highlight the selected 68 candidates. The periodic vertical "stripes" of events are primarily because the trigger threshold is around the noise level with maximum Galactic noise. The events are fewer after March due to incomplete data.

\section{Ongoing and Future Work}

The analysis will be improved by 1) reducing the errors in both the timing offset of every readout channel and spatial position of the four LPDA for more precise reconstruction, 2) including the deconvolution procedure in the reconstruction, which is especially important for events arriving from low elevation angles, 3) improving the antenna response model to include the asymmetric characteristics. In addition, the cosmic ray measurement will also implement the approach developed for the ARIANNA stations [7], but relies on accurate modelling of antenna gain and needs to take reflective effects into account. We plan to search for coincidences between HCR and HRA stations.

In next season (around November 2017), the bandwidth of the LPDA will be increased from 
140-500 MHz to $100-500 \mathrm{MHz}$ to increase the sensitivity to cosmic rays. In addition, a new HCR station will be deployed with 8 LPDAs on three separate towers. With more channels and extended separation between receivers, significantly better angular resolution is expected.

\section{Acknowledgements}

We wish to thank the US National Science Foundation for its support of the ARIANNA project under grant PHY-1607719, support from National Research Nuclear University MEPhI (Moscow Engineering Physics Institute), from the German Research Foundation (DFG, grant NE 2031/11), and for the outstanding cooperation in field planning and operations by the Antarctic Support Contractor. The project is also supported by the pioneer program of Ministry of Science and Technology (MOST) in Taiwan.

\section{References}

[1] T. Huege, Radio detection of cosmic ray air showers in the digital era, Phys. Rep. 620 (2016) 1.

[2] F. Schröder, Radio detection of cosmic-ray air showers and high-energy neutrinos, Prog. Part. Nucl. Phys. 93 (2017) 1 [astro-ph/1607.08781].

[3] T. Huege and D. Besson, Radiowave Detection of Ultra-High Energy Neutrinos and Cosmic Rays, [astro-ph/1701.02987].

[4] Pierre Auger collaboration, Measurement of the Radiation Energy in the Radio Signal of Extensive Air Showers as a Universal Estimator of Cosmic-Ray Energy, Phys. Rev. Lett. 116 (2016) 241101.

[5] S. Buitink et al., Method for high precision reconstruction of air shower $X_{\text {max }}$ using two-dimensional radio intensity profiles, Phys. Rev. D 90 (2014) 082003 [astro-ph/1408 . 7001 ].

[6] H. Schoorlemmer et al., Energy and Flux Measurements of Ultra-High Energy Cosmic Rays Observed During the First ANITA Flight, Astropar. Phys. 77 (2016) 32 [astro-ph/1612 . 04473 ].

[7] S. Barwick et al., Radio detection of air showers with the ARIANNA experiment on the Ross Ice Shelf, Astropar. Phys. 90 (2017) 50 [astro-ph/1506.05396].

[8] A. Nelles for ARIANNA collaboration, Cosmic-ray capabilities of the ARIANNA neutrino experiment, PoS(ICRC2017)399.

[9] J. Nam, Feasibility of antenna array experiment for Earth skimming tau-neutrino detection in Antarctica, PoS(ICRC2017).

[10] S. Barwick et al., Design and Performance of the ARIANNA Hexagonal Radio Array Systems, IEEE Trans. Nucl. Sci. 62 (2015) 5 [astro-ph/1410. 7369].

[11] S. Kleinfelder, E. Chiem, T. Prakash, The SST Multi-G-Sample/s Switched Capacitor Array Waveform Recorder with Flexible Trigger and Picosecond-Level Timing Accuracy, [phys ics / 1508 . 02460].

[12] P. Gorham et al., Antarctic Surface Reflectivity Measurements from the ANITA-3 and HiCal-1 Experiments, J. Astron. Instrum. 6 (2017) 1740002 [astro-ph/1703. 00415 ].

[13] J. Nam for the ANITA collaboration, Overview of the Fourth Flight of the ANITA Experiment, PoS(ICRC2017).

[14] A. Romero-Wolf et al., An Interferometric Analysis Method for Radio Impulses from Ultra-high Energy Particle Showers, Astropar. Phys. 60 (2015) 72 [astro-ph/1304. 5663]. 